\title{
A case where access implies qualia?
}

\section{ANDY Clark}

Block (1995) famously warns against the confusion of 'access-consciousness' and 'phenomenal consciousness'. Access consciousness occurs when the content of a mental state is poised for the control of rational action, for verbal report and for use in reasoning. Phenomenal consciousness, by contrast, involves the harder-to-define presence of experiential properties, of there being 'something it is like' to see red, to hear a distant bell, and so on. It is the explanation of phenomenal consciousness that constitutes the 'hard problem' of consciousness highlighted in Chalmers 1996. Block, like Chalmers, believes that many attempted explanations of phenomenal consciousness are really just explanations of (various forms of) accessconsciousness, and that the two notions are conceptually quite distinct (Block 1995: \$3, Chalmers 1996: ch. 1). I shall argue, however, that there is at least one kind of case in which facts about access seem to imply the presence of full-blown phenomenal consciousness - a kind of case, that is, in which given the facts about access it is impossible to conceive of the absence of phenomenal consciousness.

Consider a system (being, organism, whatever) capable of perceptually detecting a variety of differences between stimuli. And suppose also that this system can be interrogated about its own acts of perceptual differencedetection. Take a particular incident in which the system detects, for example, the colour difference between two visually presented stimuli. Interrogated about this act of detection, the system must (I suggest) say one of two things. It must say either:

(a) I have no access to the act by means of which I detect the difference. The answer just comes to me. I perceive nothing when I make my judgements - I simply find myself saying that there are two objects, one red and one yellow, and so on.

Or:

(b) I have access not just to the products of my sensory activity, but also to certain aspects of the sensory activity itself. For example, I am non-inferentially aware that I am using a visual rather than a tactile modality. I am aware that I see, rather than hear or feel, the difference.

But in that case it must say that there is something it is like to see the difference rather than e.g. to smell it. For in what else could direct introspective (non-inferential) access to the modality consist? To be access- 
aware of the act of detecting a difference requires at least saying (honestly) that the two items seem different in some modality-specific respect. So in this case, access-awareness (of the act of detecting a difference using a specific modality) seems to imply that there is (or is reported to be) something it is like to detect the difference.

This little argument, if correct and non-question begging (see below), suggests that pace Block and Chalmers there may very well be genuine conceptual links between specific patterns of access and report and phenomenal consciousness. The argument shares much of the flavour of Dennett's various attempts to explicate phenomenal consciousness in terms of patterns of access (Dennett 1991, 1993). But rather than focusing broadly on the notion of widespread and sustained availability for report and control, it stresses the bridging role of a specific kind of report and access viz. access to acts of detection rather than to information about states of affairs detected. It is also clearly related to accounts that stress 'self-monitoring' (e.g. Lycan 1997) though it does not need to involve any kind of higher-order thought theory. Knowing about an act of detection is a 'first order' phenomenon, on a par with knowing about the world. It is not strictly knowledge about knowledge, or thinking about thinking. Nor is the verbal reportability of the act of detection necessary for or constitutive of the presence of such access. What matters is just that the act by means of which the difference is detected is non-inferentially marked, for the creature in question, as involving (for example) a visual modality. (Notice that this is precisely the test used by Cowey and Stoerig (1995), to convince us of genuine blindsight in a monkey: the monkey was trained to touch a screen in response to a visually presented target. When forced to respond to a target presented in the blind hemifield, the monkeys often succeeded. Yet they also indicated (by touching a different part of the screen) that on these forced trials they judged there to be no visual target present. The monkeys thus denied the visuality of their access to these targets).

Interestingly, Chalmers himself toys with a related argument (1996: 287-92) but backs off at the critical moment. Chalmers here asks how the process of perception is likely to strike a well-designed intelligent agent. $\mathrm{He}$ suggests that in all likelihood the system will have no real access to the computations or processes at work when, for example, visual input is used to yield a judgement like 'there is a red cup on the table'. As a result, it faces a problem when challenged. If someone asks 'how do you know that?' there is, in a certain sense, 'nothing it can say over and above 'I just know, directly' (290). A plausible result, according to Chalmers, is that it will form the idea that the visually perceived differences are 'brutely and primitively different, differing in their 'quality' (290). Chalmers concludes, very revealingly, that: 
Given this kind of direct access to information states...it is natural to expect the system to use the language of 'experience' and 'quality' to describe its own cognitive point of view on perception. And it is unsurprising that this will all seem quite strange to the system: these immediately known, ineffable states which seem so central to its access to the world, but which are so hard to pin down. Indeed, it is natural to suppose that this would seem odd to the system in the same sort of way in which consciousness seems odd to us.' (Chalmers 1996: 291)

In my view, Chalmers is here exceedingly close to a partial dissolution of the 'hard problem' itself: close to showing that certain facts about patterns of access (the 'easy' stuff) can actually imply facts about phenomenality and 'what it's like'. It is instructive, therefore, to see what - supposedly goes wrong, and in what ways our own argument differs. Two points seem especially relevant.

First, Chalmers is imagining a case in which the intelligent system has no access whatsoever to the processing underlying its judgements. But as he himself notes (291), this leaves it less than clear why the system's perception-based judgement would not strike it as involving something more like blindsight, i.e. why it could not report that the judgement just came to it, without the positing of a mysterious 'qualitative difference'. By contrast, the case we imagined turns on a degree of genuine, if partial, access to the underlying processing - enough, at a minimum, to correctly identify the modality underlying the judgement as visual (or whatever).

Second, and relatedly, the obvious response from the fans of the phenomenal is that since we are still operating at the level of judgements (typically verbal reports) we cannot infer the presence of 'real qualia'. Our argument, it will be said, 'establishes at most that a certain kind of access implies a certain tendency to judge and report a difference (the sort of tendency my zombie twin might have) rather than implying a real phenomenal difference' (Chalmers, personal communication). All we have done, it is thus argued, is explained why we think there are qualia, not why there are qualia.

This, I think, is the crucial issue upon which so much in this literature turns. Do we really need to do more than explain why we sincerely judge there to be qualia? (Dennett is perhaps the prime example of someone who thinks that explaining that is, indeed, explaining consciousness, hard problem and all - see e.g. Dennett (1991)). I suggest that we do need to do more - but not much more! What we need to do is to show how these sincere judgements can be true. But I think we have made a start on this. We have seen, for example, that part of what is involved is direct, non-inferential access to the type of modality underlying the judgement. Since someone might think they have such access and actually lack it, or think they lack it and actually have it, we have a way of driving a wedge between honest 
report and truth - something essential, I concede, to a realist (rather than eliminativist) use of the kind of argument advanced earlier. There is thus something more to our story than just a pattern of judgements. There is a pattern of actual (ultimately neurophysiological) access which is underwriting the judgements: restricted access to enough aspects of the process leading to judgement to veridically mark the access as visual, or whatever.

Is this enough to make a judgement about qualitative feel true? Chalmers must say it isn't, that his 'zombie twin' can make the same judgements, utilizing the appropriate internal access paths, yet still lack genuine phenomenal awareness. I claim this is strictly inconceivable: honest reports of genuine direct, non-inferential access to acts of perceptual difference detection imply the presence of genuine phenomenal differences. A being so organized and functioning must report phenomenal differences, and there is a further fact of the matter that makes such reports typically true. To say that this is insufficient because that further fact is not itself a phenomenal fact, but a fact about access, is to beg the question against any account which does not acknowledge brute phenomenal facts: it is to make the reductive - or access-based - explanation of phenomenal consciousness impossible by stipulation. I see no reason to accept such a constraint.

To be convincing, however, there are several further issues that need to be dealt with. I shall close by mentioning four of them.

The first issue concerns the pivotal notion of direct, non-inferential access to the modality involved in an act of detection. What exactly does this mean? One thing it obviously can't mean is something like 'access by phenomenal feel'. For the notion is supposed to help explain what 'phenomenal feel' is, not merely assume it. The idea, basically, is that the agent is built or designed so that its personal-level, reportable knowledge and beliefs concern not just the way the world, is, but its ways of knowing about the world: that it has some access to, and hence knowledge of, its own perceptual activity, and that this knowledge is not generated by any process of conscious, personal-level inference. The being thus knows that a given judgement is, say, visually-based without relying on any inference such as: 'If I close my eyes, I fail, so this must be visually-based'. This latter inference is available, for example, to the blindsight patient who (let's assume) lacks all visual phenomenology in the blind region while retaining the capacity to use visual information from that field to make better-thanchance judgements. By contrast, we do not normally need to engage in experiments or to deploy conscious inference to know that we are using visual information. Why? Because - presumably - we have some access not just to what information is being encoded, but to how it is being encoded, with different modalities encoding information in distinctively different ways (a notion that could be further unpacked by looking at the ease of 
usability of the differently coded information, at the patterns of inferences it supports and inhibits, and so on).

Notice, then, that direct non-inferential access, as I am using the phrase, would not occur in a system in which incoming information is simply tagged in a way that informs the system of the sensory modality involved. A robot that processes visual information and that is simultaneously provided with such a signal (meaning roughly "this information is being gathered by visual sensing") would not count as having direct access to the act of visual detection. For it has no access to the characteristic properties of the visual encoding itself: no access, that is, to the very properties in virtue of which the encoding is visual rather than, say, aural. Just what these properties are is, of course, a largely empirical matter. But there seem to be at least two substantially different ways in which the notion (of direct, non-inferential access to the modality involved in an act of detection) could be cashed. One way - the simplest - would be if the inner vehicles of (say) visual content were distinctive - were different from the inner vehicles of (say) aural content. In this vein it might be imagined that visual encodings (to stick with that example) carry a distinctive kind of spatial information and that the inner vehicles of visual content are thus distinctive in some way that relates to the kind of information they carry. Access to the modality involved in an act of detection would then involve what Güzeldere (1997: 793) calls 'perception of the representational vehicle', i.e. a kind of inner sensing of the carriers of content rather than of the contents themselves. It is, however, perfectly possible that there is nothing special or distinctive about the inner vehicles that could help distinguish one modality from another (Akins (1996: 16) depicts Dennett as roundly denying the need for distinctive inner vehicles for distinct modalities - but I shall not enter into the exegetic debate here). A second (and to my mind preferable) way to understand the kind of access involved is, however, not committed to any strong claims about the existence of distinctive inner vehicles. Instead, it would stress the different ways in which contents can be poised for the control of skilled action. The idea, which relates to ideas put forward in Evans (1985) and pursued by Grush (1996), would be that what marks information as belonging to one modality rather than another is the way it is positioned to guide skilled activity. Grush and Evans are concerned with, for example, the spatiality of certain experiences and claim that experience has spatial content precisely insofar as it allows an organism to deploy certain behavioural skills - to move the body towards a sound, to point, reach out and so on (see Evans 1985: 389, and discussion in Grush 1996). (Notice that the idea is not that the organism need actually deploy these skills - indeed, it may be injured or paralysed and unable to do so. What matters is that the experience present itself to the 
creature as something upon which, in good conditions, it could bring such skills to bear - see Grush 1996: para. 7). Perhaps, then, what we have access to when we have access to the modality involved in an act of detection is the specific battery of skills that we could have deployed. Insofar as the sets of skills differ according to the modality involved (a plausible supposition, it seems to me), access to the sets of skills which could have been deployed would constitute direct non-inferential access to the modality in use - and would do so without committing us to the presence of, or detection of, distinctive inner vehicles.

The second issue concerns the opening move of the argument. David Chalmers and Jesse Prinz (personal communications) both wonder whether someone could challenge our story by attacking the early disjunction which states that either a perceptual judgement that $\mathrm{P}$ presents itself as 'just coming' to an agent (like blindsight 'hunches') or as being grounded in appreciation of a phenomenal difference. What, Chalmers asks, about simple beliefs? If you ask me, do I believe that London is the capital of England, I'll say yes; but this isn't a hunch, nor is it mediated by some distinctive phenomenal feel. Here, then, we seem to confront cases of conscious belief that are not associated with any modality-specific kind of phenomenal feel, and that do not seem well-described by either one of the initial disjuncts.

Such cases present interesting problems in their own right. But they do not impact the present argument. For the opening disjunction explicitly limits itself to the case of perceptual judgements. The claim is that in this special case, access to the act of (perceptual, modality-specific) detection forces the system (assuming it is intelligent enough to be thus interrogated) to posit the presence of modality-specific phenomenal differences: forces it to judge - like Cowey and Stoerig's monkeys - that it actually sees (or hears, or feels) differences between stimuli. Perhaps the cases of the bare attitudes (the belief that Clinton is president, etc.) show that conscious awareness does not always involve phenomenal feel. Or perhaps they show that not all phenomenal feel is modality-specific. Or (most in line with the present account) that consciously believing that $\mathrm{P}$ does feel like something, because it involves access either to the distinctive properties of a certain kind of (non-perceptual) inner encoding, or to a distinctive set of deployable skills. But whatever the lesson, our special case argument is unaffected.

The third issue concerns explanatory targets and methodology. A common reason for rejecting attempts to account for phenomenal consciousness in terms of judgement is that phenomenal consciousness is supposed to explain the patterns of judgement, and so cannot be constituted by them. A related issue (raised in Chalmers 1996: 187) concerns the role of phenomenal consciousness as an explanandum. You can't explain 
it away by explaining patterns of judgement, because it is the thing itself (the first-person experience of phenomenal content) that needs to be explained, not the judgements. The contrast here is with cases like belief in God, where (arguably) it makes sense to suppose that perhaps the judgements that God exists, etc. are all that need to be explained.

The response to these legitimate concerns is, again, to notice that, according to our story, the judgements are not everything. They are merely indicative of cognitive access to acts of perceptual detection. It is this access that is posited as the reductive basis of perceptual phenomenology. The phenomenal consciousness thus really does cause the (veridical) reports, and what we (reductively) explain is first person perceptual experience. (Notice also that popular candidates such as widespread availability for the control of action, 'cerebral celebrity' (Dennett 1993) and the like are not - on this account - themselves the reductive correlates of phenomenal consciousness. What counts is non-inferential personal level access to acts of detection. As it happens, personal level access to anything seems to help position that item for the widespread control of action. But this positioning is secondary and non-essential on the account on offer).

The fourth (and final) issue again concerns exactly what the story explains. But this time, the question is not 'does it explain phenomenal consciousness at all?' but 'does it explain why red (or whatever) looks like that, or only, (at best), that it looks like something?' Here, we must be humble. The argument I have sketched shows only (at best) that given access to the act of perceptual detection, it must look like something when, for example, we judge that one cup is red and the other green. Why, then (as Chalmers is again likely to ask - see e.g. Chalmers 1996: 188) does it look like this? Our story doesn't say. What it does show (I claim) is that it had to look (phenomenally look) like something. And this - if it is true is surely a significant fact. Compare this situation: someone shows that given certain facts, (perhaps about the conservation of energy and the persistence of matter), the waters from a broken dam had to go somewhere. This does not explain why the waters went just where they did that is, in a way, an accident of local topography and geology. But just knowing they had to go somewhere makes it immediately less mysterious that they ended up where they did! I don't claim this is an exact parallel. But I do believe that once we see why there is phenomenal experience at all, the task of explaining why it is like this rather than like that must become somewhat more tractable.

Plenty of other questions must be left in the air. The argument as sketched applies only to cases of sense-based perceptual experience. What about the rest of phenomenal consciousness? What of moods, itches, pains and the poignant longing for a cold cocktail? Perhaps the story could be 
extended to include access to acts of detection in which the modality is broadly proprioceptive and the act is one of sensing states of the body (Damasio 1994 presents some ideas that might fit in here). What about the powerful empirical links (Mack and Rock 1998) between perceptual awareness and attention? Here, the fit is potentially very good: it might well prove to be the case (though it need not) that some form of attention is necessary (see also Milner and Goodale 1995: ch. 7) for personal-level access to an act of detection. But these are all niceties for a future occasion. The point, to close, is simply this: that certain types of access (what I am calling access to acts of detection) seem to literally force an intelligent system into phenomenal space. They mark out a necessarily zombie-free zone. Could this be a place to anchor a scientific account of phenomenal consciousness? ${ }^{1}$

Washington University St. Louis, MO 63130, USA andy@twinearth.wustl.edu

\section{References}

Akins, K. 1996. Lost the plot? reconstructing Dennett's Multiple Drafts theory of consciousness. Mind and Language 11: 1-43.

Block, N. 1995. On a confusion about a function of consciousness. Behavioral and Brain Sciences 18: 227-47.

Chalmers, D. 1996. The Conscious Mind. New York: Oxford University Press.

Cowey, A. and P. Stoerig. 1995. Blindsight in monkeys. Nature 373: 247-49.

Damasio, A. 1994. Descartes’ Error. Putnam, NY: Grosset.

Dennett, D. 1991. Consciousness Explained. New York: Little Brown \& Co.

Dennett, D. 1993. The message is: there is no medium. Philosophy and Phenomenological Research 53: 919-31.

Evans, G. 1985. Molyneux's question. In The Collected Papers of Gareth Evans. Oxford, Oxford University Press.

Grush, G. 1998. Skill and spatial content. Electronic Journal of Analytic Philosophy 6: 1998 (http://www.phil.indiana.edu/ejap/).

Lycan, W. 1995. Consciousness as internal monitoring. In N. Block, O. Flanagan and G. Guzeldere, eds, The Nature of Consciousness. Cambridge, Mass: MIT Press.

Mack, A. and I. Rock. 1998. Inattentional Blindness. Cambridge, Mass.: MIT Press.

Peacocke, C. 1992. Scenarios, concepts and perception. In T. Crane, ed., The Contents of Experience. Cambridge: Cambridge University Press, 105-35.

1 Thanks to David Chalmers for critical feedback and for encouraging me to have a go at this. Thanks also to Jesse Prinz, Terry Dartnall, Jim Stone and Pete Mandik for invaluable discussion and criticism. They may not buy the argument, but they have certainly made it better. 